\title{
Demografía histórica de AnadenANTHERA COLUBRINA VAR. CEBIL (Leguminosae) en Argentina
}

\author{
MARÍA EUGENIA BARRANDEGUY1,2,3,", DARIEN E. PRADO ${ }^{3,4}$, ALEJANDRA L. \\ GONCALVES ${ }^{1,2,3}$ y MARÍA V. GARCÍA ${ }^{1,2,3}$
}

\begin{abstract}
Summary: Historical demography in Anadenanthera colubrina var. cebil (Leguminosae) from Argentina. Anadenanthera colubrina var. cebil is a South American native tree species which characterizes the Seasonally Dry Tropical Forests (SDTFs). These forests are in disjunct patches along the Neotropics. The influence of past climatic fluctuations on the current distribution of these forests deserves several interpretations because the tropical climatic conditions were unstable during Cenozoic. The aim of this study is identify traces of demographical-historical events on current patterns of chloroplastic genetic variation in Argentinean natural populations of $A$. colubrina var. cebil in order to make inferences about the temporal development of these forests. Population genetic and demographic analyses from genetic variation in three chloroplastic loci were performed. The current distribution of chloroplastic haplotypes of A. colubrina var. cebil shows clear traces of historical fragmentation between the nuclei of these forests. The Andean Piedmont nucleus maintains traces of later expansion after it arrives to the region, whereas the Misiones nucleus shows historical stability.
\end{abstract}

Key words: Anadenanthera colubrina var. cebil, cpSSRs, expansion, fragmentation, SDTFs.

Resumen: Anadenanthera colubrina var. cebil es una especie nativa sudamericana que caracteriza a los Bosques Secos Estacionales Neotropicales, los cuales se presentan en parches disyuntos dispersos a lo largo del Neotrópico. La influencia de las fluctuaciones climáticas del pasado sobre la distribución actual de dichos bosques ha merecido diferentes interpretaciones, debido a que se postula que las condiciones climáticas tropicales no fueron estables durante el Cenozoico. El objetivo de este estudio es identificar rastros de eventos demográfico-históricos sobre los patrones contemporáneos de la variación genética cloroplástica en poblaciones naturales argentinas de A. colubrina var. cebil, al efecto de hacer inferencias acerca del desarrollo temporal de estos bosques en nuestro país. Se realizaron análisis genético poblacionales y demográficos a partir de la variación genética identificada mediante tres loci microsatélites cloroplásticos. La distribución contemporánea de haplotipos cloroplásticos de $A$. colubrina var. cebil retiene rastros de la fragmentación histórica entre los núcleos de estos bosques, mientras que el núcleo Pedemontano Subandino retiene rastros de una expansión posterior a su llegada a la región en tanto que el núcleo Misiones presenta estabilidad histórica.

Palabras clave: Anadenanthera colubrina var. cebil, cpSSRs, expansión, fragmentación, SDTFs.

${ }^{1}$ Departamento de Genética. Facultad de Ciencias Exactas, Químicas y Naturales. Universidad Nacional de Misiones; Posadas 3300 Misiones, Argentina

2 Instituto de Biología Subtropical Nodo Posadas (UNaM - CONICET)

${ }^{3}$ Consejo Nacional de Investigaciones Científicas y Técnicas (CONICET - Argentina)

${ }^{4}$ Cátedra de Botánica, Facultad de Ciencias Agrarias, Universidad Nacional de Rosario. IICAR (UNR-CONICET). Campo Experimental Villarino, S2125ZAA Zavalla, Santa Fe, Argentina.

* Correo electrónico: ebarran@fceqyn.unam.edu.ar, euge_barra@hotmail.com Teléfonos: +54 3764437023 Fax: +54 3764425414 


\section{INTRODUCCIÓN}

La estructuración de la variación genética no es consecuencia únicamente de procesos microevolutivos, sino que también existen patrones resultantes de eventos demográfico-históricos tales como expansión, fragmentación y cuellos de botella poblacionales. Los efectos genéticos de la fragmentación son: pérdida de diversidad genética, aumento de la deriva genética y de la endocría y reducción de los niveles de flujo génico entre las poblaciones, siendo estos efectos consecuencias de la reducción del tamaño poblacional y del incremento del aislamiento espacial de las poblaciones (Young et al., 1996). En relación a los efectos genéticos de la expansión, en general se espera que las poblaciones en crecimiento presenten menor deriva genética que las poblaciones en estado estacionario y, por lo tanto, presenten frecuencias alélicas relativamente estables (Excoffier et al., 2009). Las poblaciones en expansión pueden presentar también un exceso de alelos raros y de mutaciones a baja frecuencia como así también un exceso de homocigosis en relación al número observado de alelos (Excoffier et al., 2009). Sin embargo, la observación de estos patrones en poblaciones que han experimentado expansión dependen de los niveles de flujo génico antiguo y reciente entre las subpoblaciones (Ray et al., 2003).

Anadenanthera colubrina (Vell.) Brenan var. cebil (Griseb.) Altschul (Cialdella, 2000) (Leguminosae, Mimosoideae) es una especie forestal nativa de América del Sur que caracteriza a los Bosques Secos Estacionales Neotropicales (Seasonally Dry Tropical Forests - SDTFs), distribuidos en el centro del continente (Prado, 2000). Esta especie es conocida como "curupay" en el Noreste Argentino, mientras que en el Noroeste se conoce como "cebil colorado" o "vilca" según su nombre quechua. Se ha determinado que la principal especie psicoactiva consumida en el área Centro Sur Andina fue A. colubrina var. cebil, presente en el registro arqueológico tanto del Noroeste Argentino como del Norte Grande de Chile (Gili et al., 2016). En Argentina, la distribución de esta especie es disyunta y comprende a las provincias fitogeográficas Paranaense y Yungas (Cabrera, 1976; Cialdella, 2000). Prado y Gibbs (1993) propusieron tres grandes núcleos de distribución para esos bosques secos, en los cuales $A$. colubrina var. cebil es la especie más paradigmática: 1) el núcleo Caatinga en el Nordeste de Brasil, donde su presencia es frecuente; 2) el núcleo Misiones a lo largo del sistema fluvial ParaguayParaná, donde es común sin llegar a ser una especie dominante y 3) el núcleo Pedemontano Subandino en el Noroeste de Argentina y Suroeste de Bolivia donde la distribución de esta especie es muy importante en su mitad sur. Los SDTFs se presentan en parches disyuntos dispersos a lo largo del Neotrópico formando un arco alrededor de la cuenca Amazónica (Pennington et al., 2000). La vegetación en estos bosques es fundamentalmente decidua durante la estación seca y está dominada por árboles y arbustos entre los que predominan Leguminosas y Bignoniáceas (Pennington et al., 2000). Recientemente, Mogni et al. (2015) propusieron al núcleo Chiquitanía en el sudeste de Bolivia y frontera con Paraguay como cuarto núcleo de distribución de los SDTFs.

La influencia de las fluctuaciones climáticas del pasado sobre la distribución actual de los SDTFs en Sudamérica ha merecido diferentes interpretaciones, debido a que se postula que las condiciones climáticas tropicales no fueron estables durante el Cenozoico, con alternancia de fases climáticas secas y frías con otras húmedas y cálidas, especialmente durante el Pleistoceno (Damuth \& Fairbridge, 1970; Ab' Sáber, 1977). Prado \& Gibbs (1993) postularon la existencia del llamado Arco Pleistocénico para intentar explicar la distribución fragmentada de estos bosques. Esta hipótesis plantea que su distribución disyunta actual representa remanentes fragmentarios de una formación boscosa cuya distribución habría sido más extensa y continua durante los ciclos secos y fríos del Pleistoceno. De este modo, en el Pleistoceno tardío podría haber ocurrido una sustitución de los tipos de vegetación predominantes como consecuencia de la contracción y fragmentación de los bosques húmedos y de la expansión de los bosques secos y viceversa. Pennington et al. (2000) contribuyen a la hipótesis del Arco Pleistocénico señalando la presencia de especies propias de los SDTFs en la Selva Amazónica, con lo cual sugieren que estas especies podrían haber colonizado la cuenca Amazónica durante el Pleistoceno. La explicación alternativa a esta amplia distribución continental de los SDTFs requiere de eventos raros y coincidentes de dispersión a larga distancia (Mayle, 2004).

Por su parte, Werneck et al. (2011) empleando 
modelos de paleodistribución y validación palinológica, establecieron una probable distribución estable de los SDTFs durante el último evento máximo glaciar (Last Glacial Maximum, LGM), seguida de una expansión hacia el sur (Núcleos Pedemontano Subandino y Misiones) que se inició en el Holoceno temprano. Basándose en este modelo, estos autores proponen cuatro refugios potenciales de estabilidad actual y a largo plazo: un refugio al sureste del estado de Mato Grosso y los núcleos Misiones, Chiquitanía y Caatinga. En una revisión reciente del estado actual de la teoría del Arco Pleistocénico, se refuerza el concepto de los SDTFs como una entidad fitogeográfica independiente, presentando además un notorio incremento en el número de registros correspondientes a los géneros y especies de leguminosas características o endémicas (Mogni et al., 2015). Así, estos autores afirman que el patrón de distribución del Arco Pleistocénico original, tal lo propuesto por Prado (1991) sigue siendo claramente visible y reconocible en los mapas de distribución actuales.

El genoma cloroplástico es de utilidad en estudios de genética evolutiva, aunque la detección de variación intraespecífica a nivel de secuencias es siempre un desafío debido a la baja tasa de mutación que caracteriza a dicho genoma. Los microsatélites de ADN cloroplástico (cpSSRs) resultan marcadores útiles y muy difundidos para estudios poblacionales debido a sus elevados niveles de polimorfismo (Navascués et al., 2006).

Estudios recientes empleando marcadores microsatélites tanto del ADN cloroplástico como del ADN nuclear, identificaron presencia de estructuración genética en poblaciones de $A$. colubrina var. cebil provenientes de las provincias fitogeográficas Paranaense y Yungas (Barrandeguy et al., 2014). Además, la estructura genética cloroplástica y la representatividad fue analizada en dichas poblaciones permitiendo concluir que la elevada frecuencia de haplotipos únicos junto con los elevados niveles de fijación de los mismos, reflejarían la acción de la deriva genética tras largos períodos de aislamiento (Goncalves et al., 2014). Por su parte, Calonga Solís et al. (2014) empleando secuencias de una región intrónica del ADN cloroplástico, dataron en aproximadamente 1.300.000 años el tiempo de divergencia entre los núcleos Caatinga y Misiones y en unos 600.000 años la divergencia entre los núcleos Misiones y Pedemontano Subandino.

Aunque los marcadores cpSSRs han sido ampliamente utilizados para estudios de estructuración genética y de flujo génico mediado por semillas, el potencial de los cpSSRs para estudios demográficos ha recibido poca atención. Navascués et al. (2006) demostraron, mediante simulaciones y datos empíricos, la utilidad de los cpSSRs para la detección de expansión poblacional y para la estimación de la edad relativa de la misma. Los estudios de demografía histórica a partir de información genética se basan en la teoría coalescente (Emerson et al., 2001). En una población estable los eventos coalescentes son escasos hacia el pasado, dando una genealogía dominada por bifurcaciones antiguas con mutaciones distribuidas principalmente en las ramas entre los nodos. Contrariamente, en el caso de poblaciones con un crecimiento repentino, los eventos coalescentes ocurren principalmente durante la expansión, generando una genealogía con forma de peine donde las mutaciones son más abundantes en las ramas terminales (singletons). Como consecuencia de esto, las expansiones poblacionales son detectadas por un exceso de singletons o un exceso de haplotipos como resultado de ellos (Navascués et al., 2006).

El objetivo principal de este estudio es identificar rastros de eventos demográfico-históricos sobre los patrones contemporáneos de la variación genética cloroplástica en poblaciones naturales argentinas de A. colubrina var. cebil, al efecto de hacer inferencias acerca del desarrollo temporal de los SDTFs en Argentina.

\section{Materiales y Métodos}

Área de estudio y metodología de muestreo

Se analizaron 83 individuos adultos de $A$. colubrina var. cebil provenientes de ocho sitios de muestreo localizados en los núcleos Misiones y Pedemontano Subandino (sensu Prado \& Gibbs, 1993) de manera de cubrir el área de distribución de la especie en Argentina (Tabla 1). Se identificó taxonómicamente a los especímenes colectados por medio de los tratamientos disponibles para el género (Altschul, 1964; Cialdella, 2000; Martínez et al., 2013). Se colectaron hojas jóvenes de individuos 
Bol. Soc. Argent. Bot. 51 (4) 2016

Tabla 1. Descripción de los sitios de muestreo analizados.

\begin{tabular}{|c|c|c|c|c|c|c|c|c|}
\hline $\begin{array}{l}\text { Núcleo } \\
\text { SDTF }\end{array}$ & Sitio de muestreo & Provincia política & $\mathbf{n}$ & NA & $N E$ & $N A U$ & $\mathrm{He}$ & Georreferencia \\
\hline \multirow{3}{*}{ Misiones } & $\begin{array}{c}\text { Candelaria } \\
\text { (Cand) }\end{array}$ & Misiones & 20 & 1,33 & 1,07 & 0,33 & 0,19 & $\begin{array}{l}27^{\circ} 26 \text { '58,200" S } \\
55^{\circ} 44^{\prime} 20,220 \text { ' O }\end{array}$ \\
\hline & $\begin{array}{l}\text { Santa Ana } \\
\text { (SA) }\end{array}$ & Misiones & 16 & 1,33 & 1,29 & 0 & 0,50 & $\begin{array}{l}27^{\circ} 25^{\prime} 55,920^{\prime \prime} \mathrm{S} \\
55^{\circ} 34^{\prime} 16,680^{\prime \prime} \mathrm{O}\end{array}$ \\
\hline & $\begin{array}{c}\text { Santa Tecla } \\
\text { (ST) }\end{array}$ & Corrientes & 4 & 1,00 & 1,00 & 0 & 0 & $\begin{array}{l}27^{\circ} 36^{\prime} 45,700 " \mathrm{~S} \\
56^{\circ} 23^{\prime} 39,700^{\prime \prime}\end{array}$ \\
\hline \multirow{5}{*}{$\begin{array}{l}\text { Pedemontano } \\
\text { Subandino }\end{array}$} & $\begin{array}{l}\text { Jujuy } \\
\text { (Juj) }\end{array}$ & Jujuy & 19 & 1,33 & 1,04 & 0,33 & 0,07 & $\begin{array}{l}23^{\circ} 45^{\prime} 15,012 " \mathrm{~S} \\
64^{\circ} 51^{\prime} 12,996 " \mathrm{O}\end{array}$ \\
\hline & $\begin{array}{l}\text { Tucumán } \\
\text { (Tuc) }\end{array}$ & Tucumán & 14 & 1,00 & 1,00 & 0 & 0 & $\begin{array}{l}26^{\circ} 47^{\prime} 26,100^{\prime \prime} \mathrm{S} \\
65^{\circ} 18^{\prime} 58,140 \text { ' O }\end{array}$ \\
\hline & $\begin{array}{l}\text { Tucumán Sur } \\
\text { (Tuc S) }\end{array}$ & Tucumán & 3 & 1,00 & 1,00 & 0 & 0 & $\begin{array}{l}27^{\circ} 18^{\prime} 58,500 " \mathrm{~S} \\
65^{\circ} 45^{\prime} 16,100 \text { " O }\end{array}$ \\
\hline & $\begin{array}{c}\text { Catamarca } \\
\text { (Cat) }\end{array}$ & Catamarca & 4 & 1,33 & 1,27 & 0,33 & 0,675 & $\begin{array}{l}28^{\circ} 02^{\prime} 01,400 \text { ' } \mathrm{S} \\
65^{\circ} 41^{\prime} 55,300 \text { ' O }\end{array}$ \\
\hline & $\begin{array}{l}\text { Guasayán } \\
\text { (Gua) }\end{array}$ & $\begin{array}{l}\text { Santiago del } \\
\text { Estero }\end{array}$ & 3 & 1,00 & 1,00 & 0 & 0 & $\begin{array}{l}28^{\circ} 08^{\prime} 13,880^{\prime \prime} \mathrm{S} \\
64^{\circ} 49^{\prime} 27,580^{\prime \prime} \mathrm{O}\end{array}$ \\
\hline Total & & & 83 & $1,17^{*}$ & $1,08^{*}$ & $0,12^{*}$ & $0,18^{*}$ & \\
\hline
\end{tabular}

*valor promedio.

adultos siguiendo la metodología descripta en Barrandeguy et al. (2014). Los individuos fueron georreferenciados mediante el sistema de posicionamiento global (GPS). Las hojas se conservaron en sílica gel dentro de bolsas rotuladas de cierre hermético hasta su procesamiento en el laboratorio. Muestras de estos individuos fueron depositadas en el herbario MCNS (Museo de Ciencias Naturales de la Universidad Nacional de Salta) y en el herbario de la Cátedra de Genética de Poblaciones y Cuantitativa (Facultad de Ciencias Exactas, Químicas y Naturales - UNaM).

Polimorfismo en loci microsatélites de ADN cloroplástico (cpSSRs)

El ADN genómico total fue extraído empleando el protocolo de extracción ROSE (Steiner et al., 1995) modificado por García et al. (2007). Se amplificaron tres loci microsatélites cloroplásticos Ccmp4, Ccmp5 y Ccmp 7 mediante cebadores universales desarrollados por Weising \& Gardner (1999). Los cebadores forward de dichos loci fueron modificados mediante la incorporación de la secuencia del cebador universal M13 (18 pb) a su extremo 5 'para la posterior genotipificación de los individuos mediante el empleo del método de los tres cebadores propuesto por Schuelke (2000) modificado por Goncalves et al. (2013). El cebador universal M13 fue sintetizado incorporando una marca fluorescente $(\mathrm{FAM}=$ azul) a su extremo 5'. Las reacciones de amplificación se realizaron en un volumen final de $15 \mu \mathrm{l}$ empleando $1 \mu \mathrm{l}$ de $\mathrm{ADN} ; 1 \mathrm{X}$ de buffer $\mathrm{KCl}$; 2,5 $\mathrm{mM}$ de $\mathrm{Cl}_{2} \mathrm{Mg}$ (Thermo Scientific - Fermentas); 0,2 mM de cada dNTP (Thermo Scientific - Fermentas); 1 U de Taq polimerasa (Thermo Scientific - Fermentas); 0,97 $\mu \mathrm{Mol} ; 0,29$ $\mu \mathrm{Mol}$ y $0,39 \mu \mathrm{Mol}$ de los cebadores Reverse, M13Forward y M13-FAM, respectivamente y agua destilada estéril. Las reacciones de amplificación fueron realizadas en un termociclador con gradiente (Biometra, Göttingen, Alemania) siguiendo un protocolo de tipo Touchdown. Las condiciones de ciclado empleadas fueron: Desnaturalización inicial a $95^{\circ} \mathrm{C}$ por $4 \mathrm{~min}, 6$ ciclos de desnaturalización 
por $45 \mathrm{seg}$ a $95^{\circ} \mathrm{C}$; hibridación por $30 \mathrm{seg}$ en un rango de temperatura de $60^{\circ} \mathrm{C}$ a $55^{\circ} \mathrm{C}$ con una reducción de $1^{\circ} \mathrm{C}$ en cada ciclo y elongación por $45 \mathrm{seg}$ a $72^{\circ} \mathrm{C}$ seguidos de 23 ciclos similares empleando una temperatura de hibridación de $55^{\circ}$ C; se adicionaron 8 ciclos para la incorporación de la marca fluorescente necesaria para la posterior genotipificación. La reacción de amplificación culmina con una etapa de elongación final a $72{ }^{\circ} \mathrm{C}$ por $20 \mathrm{~min}$. La verificación de las amplificaciones fue realizada mediante electroforesis $(5 \mathrm{~V} / \mathrm{cm})$ en geles de agarosa al 1,5\% con buffer TBE $1 \mathrm{X}$ teñidos con Gel Red al $1 \%$ (Biotium) empleando un marcador de peso molecular de $100 \mathrm{pb}$ (Productos Biológicos).

El tamaño de los fragmentos fue asignado empleando el secuenciador ABI Prism ${ }^{\circledR} 3130$ (Applied Biosystems) empleando un marcador de peso estándar GS 500 ROXTM (Applied Biosystems). Para ello se utilizó el programa Peak Scanner v 1.0 (Applied Biosystems).

\section{Análisis estadístico de los datos}

A los fines de caracterizar la variabilidad genética cloroplástica se estimaron, en primer lugar, las frecuencias alélicas por locus. Además, se estimó el número promedio de alelos por locus $(N A)$, el número efectivo de alelos por locus $(N E)$ calculado como $N E=$ donde $x_{i}$ corresponde a las frecuencias alélicas (Gillet et al., 2005), el número promedio de alelos únicos $(N A U)$ y el índice de diversidad haplotípica de Nei $(\mathrm{He})$ calculado como $H e=\left\{[n /(n-1)]\left[1-\Sigma p_{i}^{2}\right]\right\}(\mathrm{Nei}, 1987)$ donde $n$ es el número de individuos analizados en una población y $p_{i}$ es la frecuencia del $i$-ésimo haplotipo en esa población. Las estimas $N A, N E$ y $N A U$ fueron obtenidas empleando el programa GenAlEx versión 6.5 (Peakall \& Smouse, 2012), en tanto que He fue obtenido mediante cálculo manual.

Se infirió la estructura genética poblacional mediante un análisis Bayesiano utilizando el programa BAPS versión 6.0 (Corander et al., 2006; Corander et al., 2008a). El modelo analizado consistió de un agrupamiento de tipo mixture (Nonspatial genetic mixture analysis) para loci ligados (Corander et al., 2008a). Mediante este análisis se determinó el número más probable de agrupamientos o clusters y se asignaron los individuos a ellos empleando únicamente información genética. El número de clusters fue tratado como un parámetro desconocido. Además, se implementó un modelo de agrupamiento espacial de individuos (Spatial genetic mixture analysis) (Corander et al., 2008b) incorporando al agrupamiento no espacial el término correspondiente a la probabilidad a priori de la posición geográfica de cada individuo. Corander et al. (2008b) demostraron que el modelo espacial mejora el poder estadístico para detectar la estructura genética poblacional cuando se dispone de un bajo número de loci. Para determinar el número de clusters que presenta la mayor probabilidad a posteriori se testaron valores de $\mathrm{k}$ clusters comprendidos en un rango de uno a ocho $(\mathrm{k}=[1-8])$ realizando 10 repeticiones para cada valor de $\mathrm{k}$.

El grado de estructuración genética poblacional se analizó mediante el índice de fijación $\mathrm{F}_{\mathrm{ST}}$ (Wright, 1965), el cual fue estimado a partir de un análisis de varianza molecular (AMOVA) según la ecuación:

$$
\mathrm{F}_{\mathrm{ST}}=\frac{\sigma_{a}^{2}-\sigma_{T}^{2}}{\sigma_{T}^{2}} \text { (Weir \& Cockerham, 1984). Para }
$$

este análisis se consideraron como poblaciones a los clusters definidos mediante el análisis Bayesiano espacial. De esta manera, los niveles jerárquicos considerados fueron: Entre regiones (entre núcleos), entre poblaciones dentro de regiones (entre clusters dentro de núcleos) y dentro de poblaciones (dentro de clusters). La significancia estadística del índice $\mathrm{F}_{\mathrm{ST}}$ fue determinada a partir de un valor de $p$ obtenido mediante 1023 permutaciones de los haplotipos dentro de grupos y entre los grupos definidos por el análisis Bayesiano, considerando al índice $\mathrm{F}_{\text {ST }}$ calculado como estadísticamente significativo cuando $p<0,05$ para el $95 \%$ de confianza. Estos análisis fueron desarrollados utilizando el programa Arlequín 3.5 (Excoffier \& Lischer, 2010).

Para determinar las relaciones filogenéticas entre los haplotipos identificados se construyó una red empleando el algoritmo Median-Joining $(M J)$ empleando el programa PopART (Leigh \& Bryant, 2015), el cual conecta a los haplotipos según su similitud genética de acuerdo a la cantidad de mutaciones presentes entre los mismos. Los haplotipos fueron determinados a partir de la combinación de los alelos, los cuales fueron identificados en pares de bases para cada locus. La red consiste en nodos y uniones que los conectan. Los nodos corresponden a los haplotipos desde el conjunto de datos o vectores medianos y las uniones 
quedan definidas por los eventos mutacionales que los diferencian. Un vector mediano es un haplotipo hipotético, el cual puede ser requerido para conectar los haplotipos dentro de la red con máxima parsimonia. Los vectores medianos representan haplotipos intermediarios ausentes ya sea porque no fueron muestreados o porque están extintos (Bandelt et al., 1999).

Para testar la posible ocurrencia de eventos de expansión histórica se implementaron las siguientes aproximaciones basadas en la teoría coalescente: la prueba de neutralidad $F_{S}(\mathrm{Fu}, 1997)$ y el estadístico $D_{\text {Tajima }}$ (Tajima, 1989), utilizando el programa Arlequin versión 3.5 (Excoffier \& Lischer, 2010). La prueba de neutralidad $F s$ se basa en el número esperado de haplotipos cuando se compara una demografía estacionaria con una de expansión. El estadístico $F s$ tomará valores negativos dentro de una población debido a un exceso de haplotipos raros (mutaciones recientes) indicando expansión poblacional (Navascués et al., 2006). La significancia estadística de la prueba fue calculada mediante 10.000 bootstraps de los datos y la obtención del estadístico Fs con un valor de $p(F s)<0,02$ se considerará evidencia de expansión poblacional. Teniendo en cuenta que $p(F s)<0,02$ equivale a $\alpha=0,05$ debido a un comportamiento particular del estadístico Fs (Fu, 1997). El estadístico $D_{\text {Tajima }}$ se calculó mediante la ecuación: $D=\pi-(\theta) / \sqrt{V[\pi-(\theta)]}$ donde, $\pi$ es el número medio de sitios que difieren entre cada par de secuencias, $V$ representa a la varianza del numerador y $\theta$ se calcula mediante la ecuación $\theta=S / a$ donde, $S$ es el número de sitios polimórficos y $a$ se calcula mediante la siguiente ecuación: $a=\sum_{i=1}^{n-1} 1 / i$ donde $i$ toma valores entre 1 y $n-1$, siendo $n$ el número de secuencias analizadas (Pybus \& Shapiro, 2009). Estos parámetros fueron estimados a partir de la distribución de las diferencias de a pares entre individuos dentro de una muestra medida como diferencias alélicas entre haplotipos cpSSRs, siendo estos datos codificados como binarios (Navascués et al., 2006). Esta distribución es afectada por la demografía de la muestra esperándose un estadístico $D_{\text {Taijma }}$ cercano a cero en una población de tamaño constante, mientras que valores negativos estadísticamente significativos indican una expansión repentina del tamaño poblacional y valores positivos indican eventos de subdivisión poblacional o cuellos de botella recientes. La significancia estadística de $D_{\text {Taijma }}$ es testada generando muestras aleatorias bajo la hipótesis de equilibrio poblacional empleando un algoritmo de simulación coalescente. El valor de $p$ para el estadístico $D_{\text {Tajima }}$ es obtenido como la proporción de estadísticos $D_{\text {Tajima }}$ aleatorios menores o iguales al observado.

\section{Resultados}

El locus Ccmp5 presentó cuatro alelos diferentes, con lo cual resultó más polimórfico que los loci Ccmp 4 y $C$ cmp 7 que presentaron tres y dos alelos, respectivamente (Figura 1). Se identificaron en los tres loci una conformación alélica propia característica de cada uno de los núcleos SDTFs analizados, en los cuales sólo el alelo 109 del locus Ccmp4 fue compartido entre los núcleos Misiones y Pedemontano Subandino. El locus Ccmp5 presentó dos alelos únicos en Jujuy y en Candelaria (133 y 139 respectivamente), mientras que el locus $C$ cmp 4 presentó un alelo único en Catamarca (110). Por su parte, los dos alelos del locus Ccmp 7 se encontraron fijados uno en cada núcleo SDTF. Cabe destacar, que los alelos únicos antes mencionados presentan marcadas diferencias en sus frecuencias entre los diferentes sitios de muestreo (Fig. 1).

Mediante la combinación de las variantes alélicas se definieron seis haplotipos (HA a HF). En la Figura 2a se presentan los sitios de muestreo en los cuales esos haplotipos fueron identificados. Las muestras analizadas no presentaron haplotipos compartidos entre los núcleos SDTFs considerados. En el núcleo Misiones el haplotipo HB es compartido entre los sitios de muestreo Candelaria, Santa Ana y Santa Tecla. En tanto, el haplotipo HC sólo está presente en Santa Ana y el haplotipo HA sólo está en Candelaria. En el núcleo Pedemontano Subandino, el haplotipo HD es compartido por todos los sitios de muestreo analizados, y los haplotipos HE y HF son únicos en Jujuy y Catamarca, respectivamente (Fig. 2a).

El índice de diversidad haplotípica de Nei $(\mathrm{He})$ reflejó niveles reducidos de diversidad genética ya que tres de los ocho sitios de muestreo considerados no presentaron variación genética cloroplástica para los loci analizados, siendo Santa Ana y Catamarca los sitios de muestreo más diversos (Fig. 3). El parámetro $N E$ tomó valores cercanos a la unidad y resultó menor a $N A$ debido a la presencia de alelos 


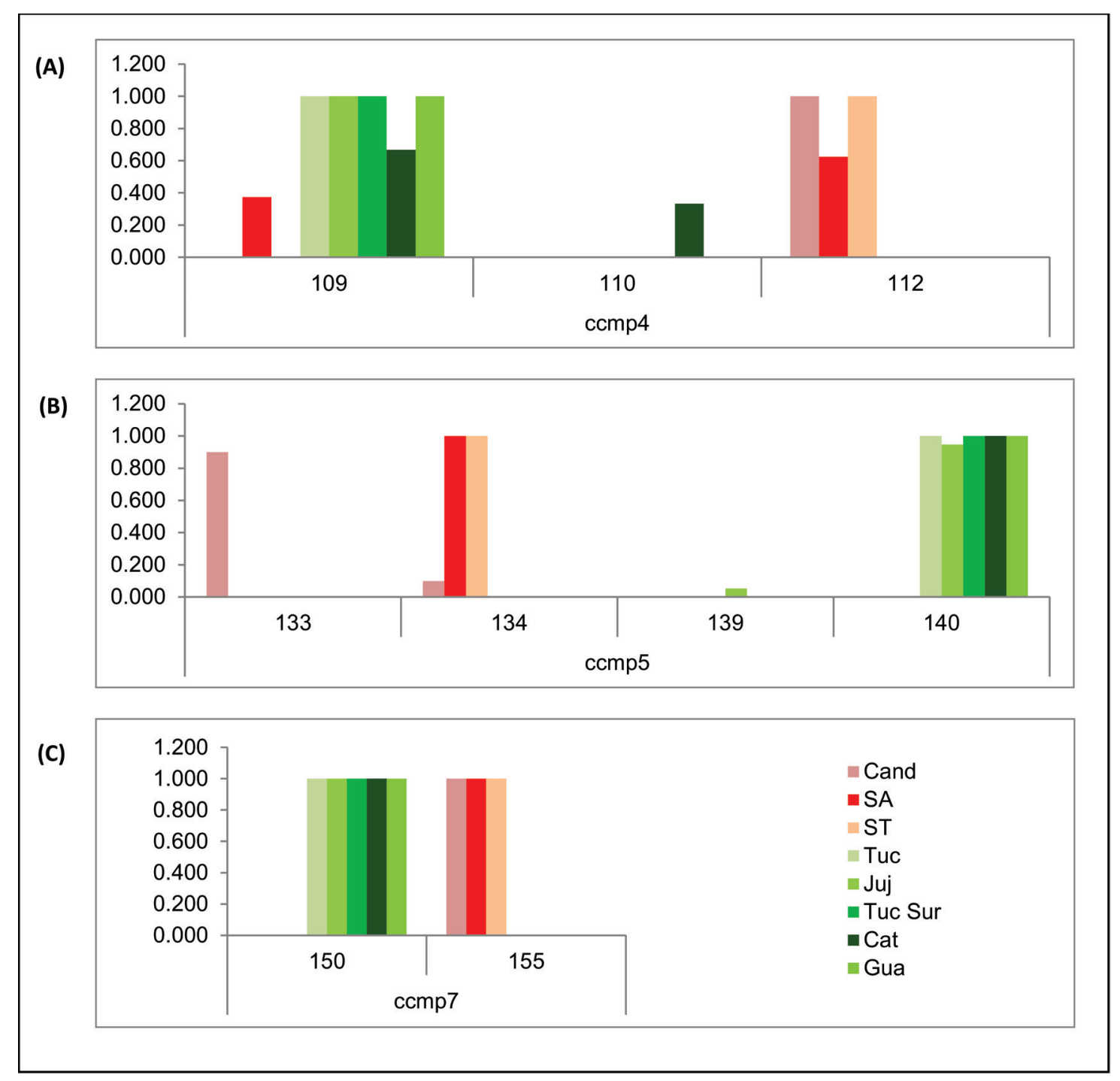

Fig. 1. Frecuencias alélicas por locus: (A) Ccmp4, (B) Ccmp5 y (C) Ccmp7. Abreviaturas de los sitios de muestreo según Tabla 1.

poco frecuentes en los sitios de muestreo. La mayor diferencia para ambos parámetros se presentó en Candelaria y Jujuy. Por su parte, Santa Tecla, Tucumán, Tucumán Sur y Guasayán presentaron NE igual a NA debido a la presencia de un único alelo en dichos sitios de muestreo (Fig. 3).

En cuanto a la determinación de la estructura genética poblacional, el modelo no espacial del análisis Bayesiano permitió establecer un número más probable de clusters igual a seis $\left(\mathrm{P}_{\mathrm{k}=6}=0,60\right)$ (Fig. 4). Por su parte, el modelo espacial estableció un número más probable de clusters de tres $\left(\mathrm{P}_{\mathrm{k}=3}=\right.$
$0,99)$, siendo los individuos asignados a los clusters 1 y 2 provenientes del Núcleo Misiones mientras que aquellos asignados al cluster 3 provienen del Núcleo Pedemontano Subandino (Fig. 4).

Mediante el AMOVA pudo detectarse que el mayor porcentaje de variación quedó contenido entre clusters dentro de núcleos $(\sim 56 \%)$, seguido por la variación entre núcleos $(\sim 38 \%)$ y finalmente el menor porcentaje de variación quedó contenido dentro de clusters ( $\sim \%$ ). El índice de fijación $\mathrm{F}_{\mathrm{ST}}$ presentó un valor estadísticamente significativo de $0,944(p<0,050)$. 


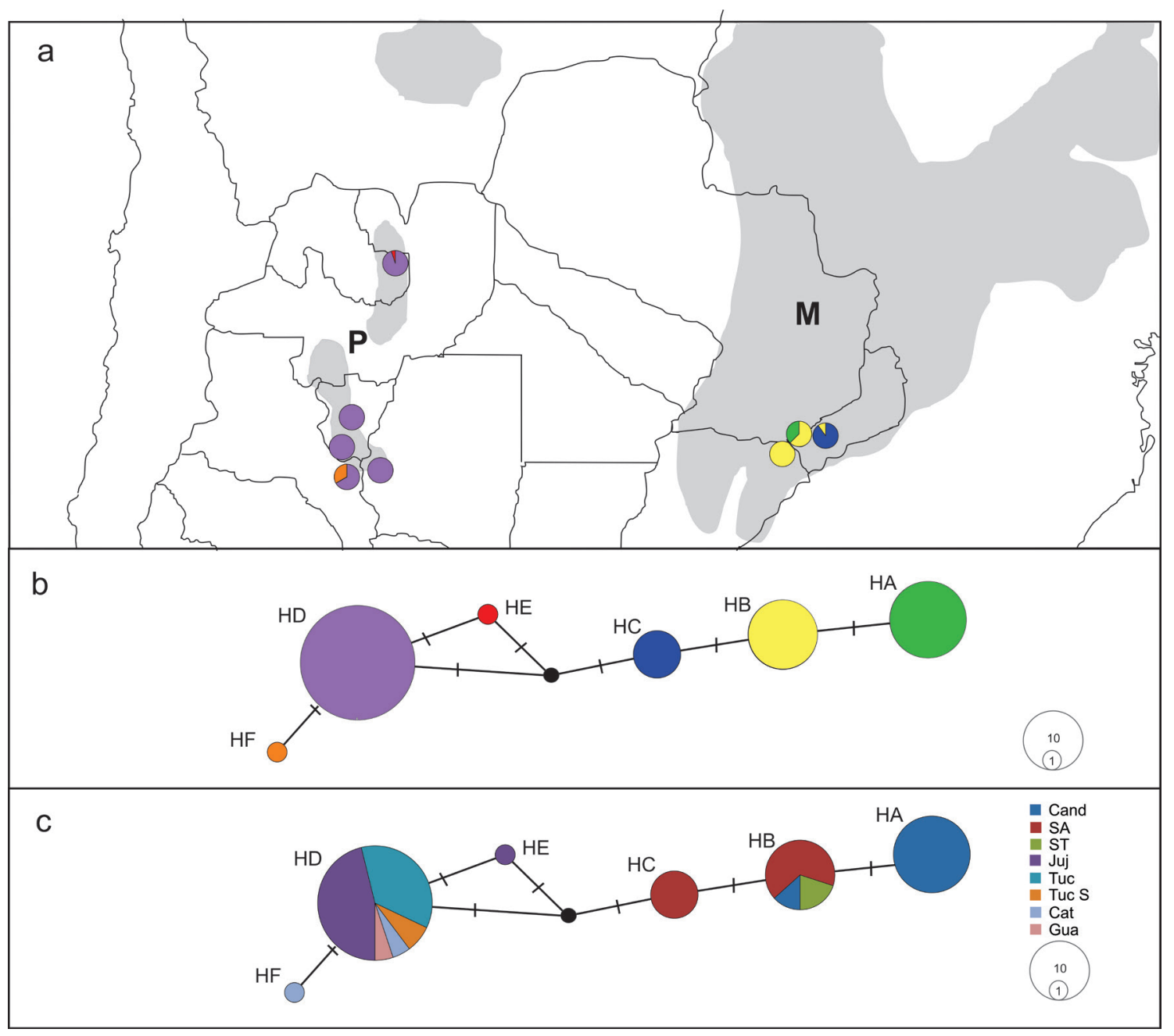

Fig. 2. a: Frecuencias y distribución geográfica de los seis haplotipos determinados mediante la combinación de las variantes alélicas (haplotipos HA a HF). En gris se indica la distribución de los Bosques Secos Estacionales Neotropicales: (M) Núcleo Misiones; (P) Núcleo Pedemontano Subandino. b: Red de haplotipos Median-Joinning. Cada círculo representa un haplotipo mientras que su tamaño representa la frecuencia. c: Red de haplotipos Median-Joinning. Los colores representan los sitios en los que fueron identificados los haplotipos. Abreviaturas de los sitios de muestreo según Tabla 1.

La red de haplotipos MJ relacionó a los seis haplotipos identificados e incorporó un haplotipo intermediario, de manera que cada par de haplotipos difiere para los alelos de un locus (Fig. 2b). El haplotipo intermediario consiste en un vector mediano necesario para obtener la mínima distancia de conexión entre los haplotipos definidos en el estudio. Además, en dicha red se evidenciaron dos grupos: el primero de ellos incluyó a los haplotipos identificados en el núcleo Misiones mientras que el segundo incluyó a los haplotipos identificados en el núcleo Pedemontano Subandino. Las muestras analizadas no presentaron haplotipos compartidos entre los núcleos SDTFs, siendo la relación entre ambos grupos incierta ya que la misma pudo ser establecida mediante un haplotipo intermediario, el cual se relaciona directamente con el haplotipo HC identificado en el núcleo Misiones y con los haplotipos HD y $\mathrm{HE}$ identificados en el núcleo Pedemontano Subandino. Cabe destacar que de 43 


\section{E. Barrandeguy et al. - Demografía histórica en A. colubrina var. cebil}

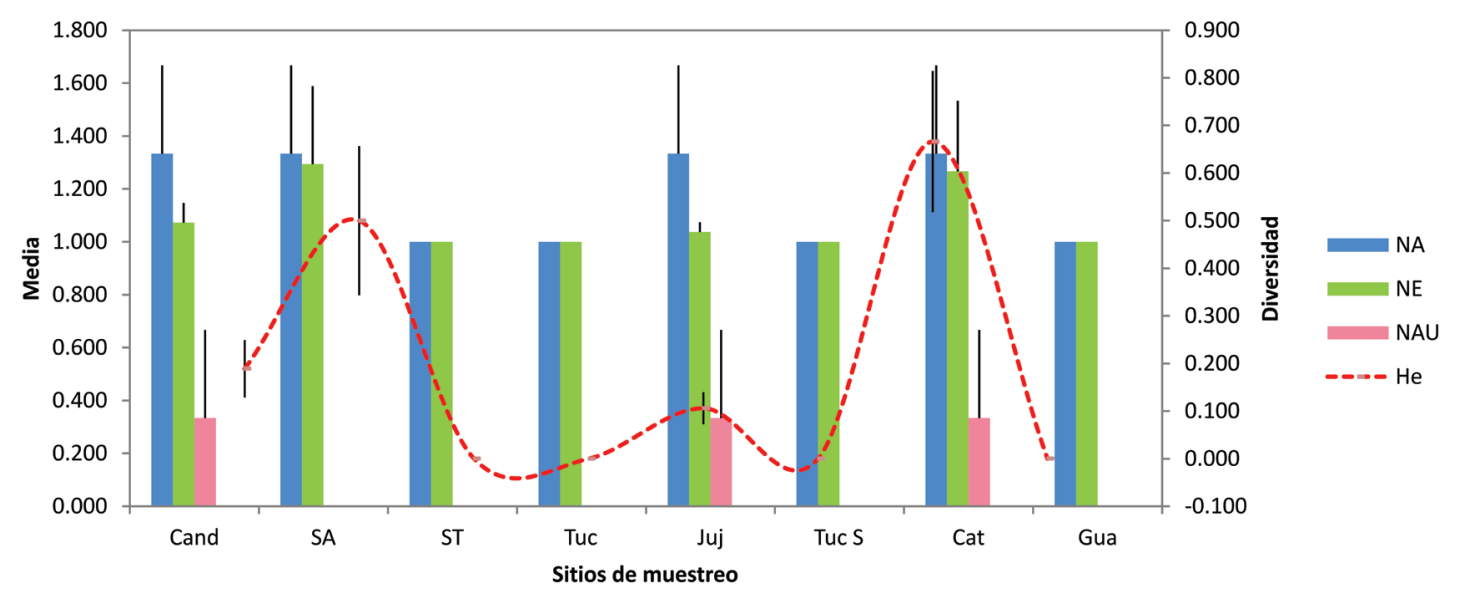

Fig. 3. Caracterización de la diversidad genética cloroplástica de los sitios de muestreo considerados (abreviaturas según Tabla 1).

individuos analizados en el núcleo Pedemontano Subandino 41 de ellos presentaron el haplotipo HD. Además, a partir de la red se evidenciaron mayores niveles de variación genética en el núcleo Misiones ya que se presentaron tres haplotipos altamente frecuentes, mientras que en el núcleo Pedemontano Subandino se presentó un único haplotipo más frecuente y ampliamente distribuido (Fig. 2c).

En cuanto a la detección de expansión poblacional, la prueba de neutralidad a partir del estadístico $F s$ evidenció expansión poblacional para el núcleo Pedemontano Subandino $[F s=$
-9,698; $p(F s)<0,02]$ mientras que para el núcleo Misiones no fue posible rechazar la hipótesis de un escenario estable $[p(F s)>0,02]$ (Tabla 2). Resultados similares fueron obtenidos a partir del estadístico $D_{\text {Tajima }}$, ya que para el núcleo Pedemontano Subandino este índice arrojó valores negativos estadísticamente significativos evidenciando una expansión poblacional en dicho núcleo $\left(D_{\text {Tajima }}=-1,877 ; p<0,05\right)$, mientras que para el núcleo Misiones el estadístico $D_{\text {Tajima }}$ resultó estadísticamente no significativo $(p<0,05)$ (Tabla 2).

No espacial

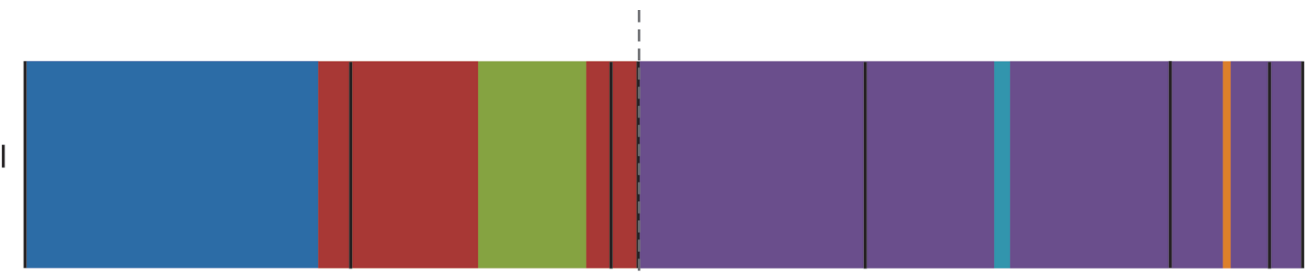

Espacial

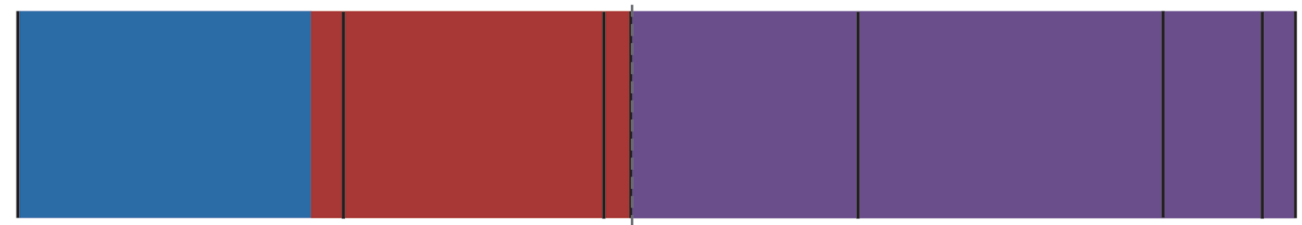

Misiones

Pedemonte Subandino

Fig. 4. Análisis Bayesiano de la estructura genética poblacional. Modelo de tipo mixture para loci ligados. 


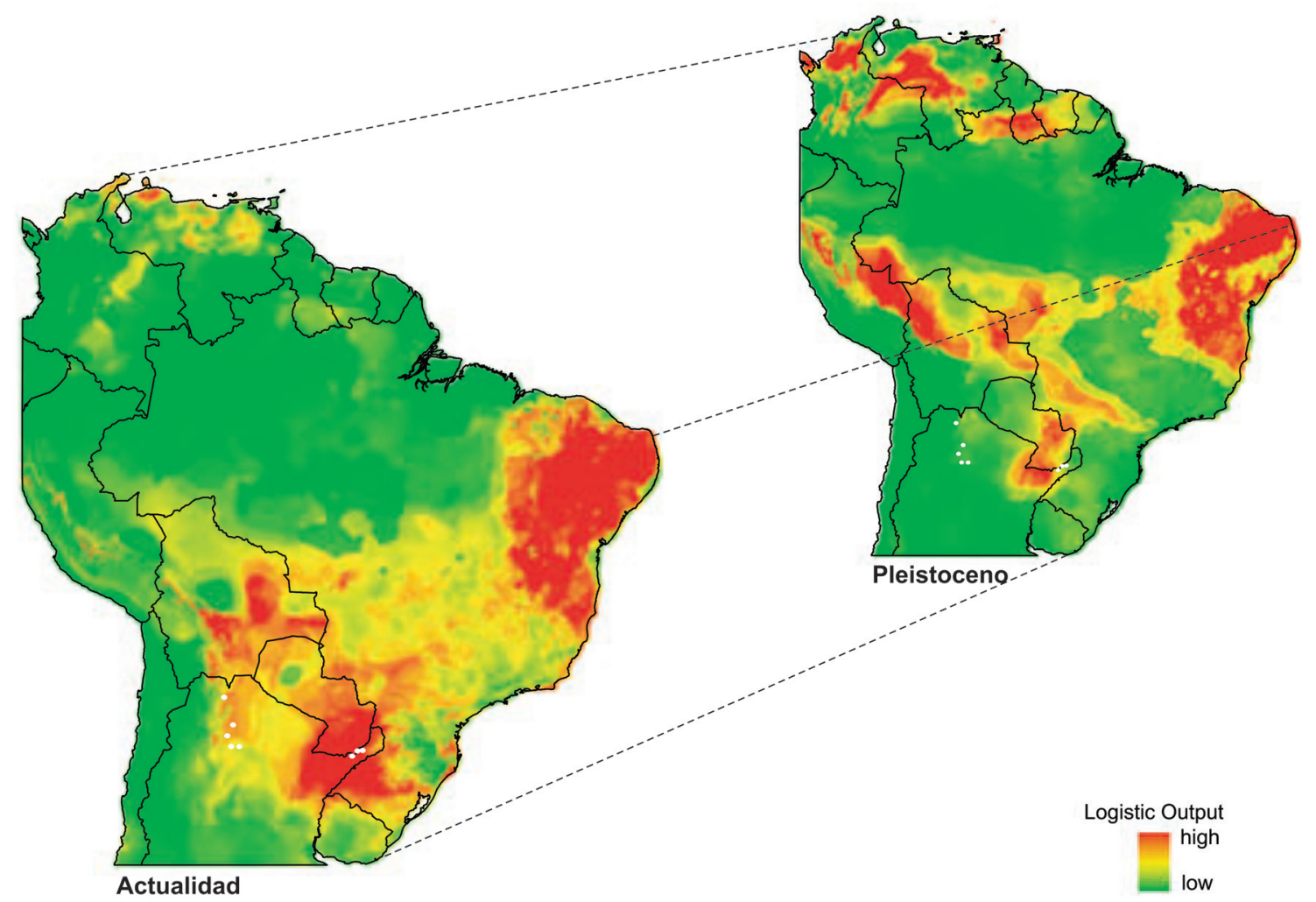

Fig. 5. Localización de los sitios de muestreo sobre los mapas de paleodistribución propuestos por Werneck et al. (2011) para el Pleistoceno y la actualidad. . En verde se representan las áreas de baja probabilidad de ocurrencia de los SDTFs, mientras que en gris claro se representan áreas estables de alta probabilidad de ocurrencia de los SDTFs. Imagen adaptada desde Werneck et al. (2011).

\section{Discusión}

La distribución disyunta actual de los SDTFs en Sudamérica y Mesoamérica fascina a botánicos y biogeógrafos (Vanzolini, 1963; Ab'Sáber, 1977; Prado \& Gibbs, 1993; Pennington et al., 2000, Pennington et al., 2009; de Oliveira et al., 2013), como así también a filogenetistas y filogeógrafos (Pennington et al. 2004; Becerra, 2005; Caetano et al., 2008) y, más recientemente, a biólogos evolutivos (Werneck et al., 2011; Collevatti et al., 2012). Aunque se ha incrementado el número de estudios filogeográficos en especies sudamericanas en el último tiempo, este número resulta aún insuficiente destacando la urgente necesidad de estudios de biogeografía histórica y de filogeografía, para comprender cómo las especies han respondido a los cambios climáticos del pasado y para predecir cómo podrán hacer frente a los cambios del presente (Turchetto-Zolet et al., 2013).

El objetivo principal del presente trabajo fue identificar evidencias de eventos demográficohistóricos sobre los patrones contemporáneos de la variación genética cloroplástica en poblaciones naturales de $A$. colubrina var. cebil, con el objeto de hacer inferencias acerca del desarrollo temporal de los SDTFs a partir de este taxón característico de dichos bosques. Prado \& Gibbs (1993) consideraron a $A$. colubrina var. cebil como el taxón clave indicador de los SDTFs debido a que representa una especie dominante o frecuente en los principales núcleos de los bosques secos de América del Sur, con excepción de aquellos de valles andinos y las costas de Colombia y Venezuela (Pennington et al., 2009), con lo cual se espera que los resultados obtenidos para esta especie puedan ser aplicados al estudio general de los SDTFs. 
Durante el Cuaternario, los cambios ambientales más relevantes han estado vinculados con cambios climáticos los cuales han impactado evolutivamente sobre las especies. Los períodos glaciares e interglaciares asociados con dichos cambios climáticos han influenciado la dinámica de la biodiversidad alterando dramáticamente la evolución de la misma y el paisaje en Sudamérica (Turchetto-Zolet et al., 2013).

Los resultados obtenidos indicaron niveles reducidos de diversidad genética en los sitios de muestreo analizados, presentando haplotipos fijados en la mitad de los mismos (Fig. 2 a y c). Debido a la organización estructural conservada del ADNcp en plantas superiores, sumado al reducido flujo génico mediado por semillas, pueden esperarse niveles reducidos de diversidad genética en este genoma (Zhan et al., 2011).

La escasa similitud genética evidenciada entre los núcleos Misiones y Pedemontano Subandino, los cuales presentan un patrón caracterizado por alelos propios y un único alelo compartido, sería consecuencia de la fragmentación y aislamiento histórico, de manera que los fragmentos boscosos actuales podrían ser vestigios de una distribución continua de los SDTFs en el pasado, tal como lo predice la Teoría del Arco Pleistocénico (Prado \& Gibbs, 1993). Ciertamente, se podría plantear la hipótesis alternativa de la dispersión a larga distancia (DLD) (Mayle 2004), para la cual ha surgido también amplia evidencia a nivel intercontinental o para especies de selvas tropicales de lluvia ( Davis et al., 2002; Lavin et al., 2004; Dick \& Heuertz, 2008; Schrire et al., 2009). Sin embargo, se ha establecido ya que resulta más parsimonioso, atendiendo al principio de la Ley de Ockham ("Frustra fit per plura quod potest fieri per pauciora"; Mautner 1997, p. 399), la hipótesis de un único fenómeno de vicariancia, que decenas de fenómenos aleatorios de DLD para un gran grupo de especies muy distantes filogenéticamente (Pennington et al., 2000; Mogni et al. 2015).

La determinación de la estructuración genética, mediante el análisis Bayesiano considerando únicamente información genética, resultó en la conformación de seis clusters, los cuales quedaron definidos cada uno por uno de los seis haplotipos identificados en los sitios de muestreo (Fig. 4). Sin embargo, pudo asociarse la estructuración genética poblacional con el origen geográfico al considerar la información espacial a priori, ya que los clusters se redujeron a tres, asignándose a dos de ellos individuos muestreados en el núcleo Misiones mientras que en el restante fueron asignados todos los individuos muestreados en el núcleo Pedemontano Subandino (Fig. 4). Dicha estructuración, a nivel de núcleos, podría ser explicada nuevamente por una fragmentación histórica de los SDTFs y el posterior aislamiento de los fragmentos.

A partir del estudio mediante secuencias de una región intrónica en $A$. colubrina var. cebil la divergencia estimada entre los núcleos Misiones y Pedemontano Subandino, en Argentina, se puede posicionar temporalmente en el Pleistoceno tardío (Calonga Solís et al., 2014) lo cual coincide con la teoría del Arco Pleistocénico propuesta por Prado \& Gibbs (1993). En cambio, TurchettoZolet et al. (2013), mediante un meta-análisis, concluyeron que los patrones filogeográficos de la biota de Sudamérica presentan una marcada estructura filogeográfica intraespecífica con múltiples linajes. Estas divisiones filogeográficas profundas indican extensos periodos de aislamiento entre las poblaciones de las especies sudamericanas $\mathrm{y}$, cuando las divisiones pudieron ser datadas, la divergencia ocurrió en el Pleistoceno medio en el $57 \%$ de las especies analizadas (Turchetto-Zolet et al., 2013).

A partir del índice de fijación $\mathrm{F}_{\mathrm{ST}}$ estimado $\left(\mathrm{F}_{\mathrm{ST}}=0,944 ; p<0,05\right)$ se determinó, según la escala establecida por Wright (1965), presencia de gran estructuración genética poblacional reflejando la fijación de haplotipos alternativos en los diferentes clusters. Los elevados niveles de estructuración genética en angiospermas se relacionan con el flujo génico mediado por semillas debido a la herencia materna del genoma cloroplástico. Así, los patrones de dispersión de semillas pueden moldear la composición y la estructura genética de las poblaciones vegetales. Especies con flujo de semillas limitado probablemente poseen heterogeneidad genética entre la descendencia de distintos individuos, mientras que especies con dispersión de semillas extensiva presentan menor estructuración genética (Hamrick et al., 1993). El movimiento de las semillas constituye el componente más importante de la dinámica de las poblaciones vegetales, ya que la regeneración y la colonización de hábitats requieren del movimiento 
de la información genética a través de las semillas (Finkeldey \& Hattemer, 2007). En este sentido, Barrandeguy et al. (2014), estudiando cuatro poblaciones de A. colubrina var. cebil determinaron moderada estructuración genética poblacional a nivel del genoma nuclear (cuya dispersión ocurre tanto por polen como por semillas) mientras que determinaron elevada estructuración genética a nivel del genoma cloroplástico e interpretaron estos resultados como consecuencia de fragmentación histórica.

El grado de estructuración genética antes analizado también se vio reflejado en la red de haplotipos (Fig. 2b), la cual representa gráficamente las relaciones entre los mismos de la manera más parsimoniosa basándose en los eventos mutacionales que los distancian. La ambigüedad del loop presente entre los haplotipos HD, HE y el haplotipo hipotético queda resuelto a partir del criterio de las relaciones interiores-exteriores. Crandall \& Templeton (1993) han demostrado que los haplotipos raros ocurren frecuentemente en las ramas exteriores y aquellos haplotipos frecuentes ocurren habitualmente en el interior. Por lo tanto, a partir de dicho criterio el haplotipo HE se conectaría con el haplotipo HD y éste se conectaría con el haplotipo hipotético no muestreado o extinto. Además, los autores previamente mencionados han propuesto un criterio geográfico para poblaciones geográficamente estructuradas mediante el cual predicen que los singletons presentan mayor probabilidad de conectarse con un alelo de la misma población que con un alelo de una población diferente. Este criterio refuerza la conexión de HE con HD ya que ambos fueron identificados en Jujuy. En la red de haplotipos se evidenciaron dos grupos integrados por los haplotipos pertenecientes a cada núcleo SDTF analizado. Los haplotipos identificados en el núcleo Misiones presentaron frecuencias moderadas y se hallaron concentrados en una extensión de aproximadamente $88 \mathrm{Km}$. La mayor variación genética contemporánea presente en este núcleo podría ser explicada bajo la hipótesis de estabilidad ancestral lo cual concuerda con lo demostrado mediante los modelos de paleodistribución de Werneck et al. (2011), quienes indicaron al núcleo Misiones como un área de estabilidad a largo plazo (Fig. 5). Por su parte, el área geográfica considerada del núcleo Pedemontano Subandino tiene una extensión de aproximadamente $550 \mathrm{~km} \mathrm{y}$, a lo largo de esta extensión, se halló un haplotipo ampliamente distribuido y dos haplotipos únicos que presentan baja frecuencia.

Debido a la evolución lenta del genoma cloroplástico, la distribución geográfica contemporánea de los haplotipos junto con las relaciones genéticas que los vinculan, pueden ser interpretadas como rastros de eventos demográficohistóricos. En Misiones se registró un haplotipo compartido de mayor frecuencia (HB) mientras que se hallaron dos haplotipos únicos de frecuencia moderada (HA y HC). Esta variación haplotípica podría representar variabilidad genética antigua propia del núcleo Misiones. Por su parte, en el núcleo Pedemontano Subandino, se registró un haplotipo a elevada frecuencia ampliamente distribuido en todos los sitios de muestreo analizados (HD). Además, en los sitios de Jujuy y de Catamarca se hallaron haplotipos únicos que presentan bajas frecuencias, considerándose a estas variantes raras como haplotipos más recientes producidos por mutación en los loci cpSSRs. Dicha distribución haplotípica en el núcleo Pedemontano Subandino sería resultado de eventos de expansión y colonización desde el Norte hacia el Sur de dicho núcleo, lo cual concuerda con los mapas de distribución propuestos por Werneck et al. (2011) donde se evidencia que el área argentina del núcleo Pedemontano Subandino corresponde a zonas inestables de baja probabilidad de ocurrencia de SDTFs durante el LGM en el Pleistoceno Superior (Fig. 5). En términos geológicos, las zonas inestables habrían sido colonizadas recientemente y por lo tanto deberían retener huellas genéticas de las expansiones poblacionales pasadas (Werneck et al., 2011). Con el objeto de testar esta consideración acerca de la distribución contemporánea de la variación genética cloroplástica de A. colubrina var. cebil se aplicaron dos pruebas de neutralidad basadas en la teoría coalescente. Los resultados obtenidos para ambas pruebas (Tabla 2) indican que las poblaciones del núcleo Pedemontano Subandino presentan elevada probabilidad de expansión poblacional, mientras que las poblaciones del núcleo Misiones presentan una historia demográfica estable. Estos resultados coinciden con las zonas de estabilidad/ inestabilidad histórica propuestas por Werneck et al. (2011) (Fig. 5). 
Tabla 2. Análisis demográficos mediante pruebas coalescentes: $F_{s}$ y $D_{\text {Telima }}$

\begin{tabular}{|ccccccc|}
\hline Núcleo SDTF & \multicolumn{3}{c}{ Fu (1997) } & \multicolumn{3}{c|}{ Tajima (1989) } \\
\hline Misiones & $\mathbf{N}$ & $\boldsymbol{F}_{\boldsymbol{s}}$ & $\boldsymbol{p}(\boldsymbol{F s})$ & $\boldsymbol{D}$ & $\boldsymbol{p}(\boldsymbol{D})$ \\
$\begin{array}{c}\text { Pedemontano } \\
\text { Subandino }\end{array}$ & 42 & 0,892 & 0,706 & 1.570 & 0,930 \\
\hline
\end{tabular}

La persistencia de algunas especies en múltiples áreas localizadas a través de su rango de distribución indica que esas especies pudieron haber persistido durante varios ciclos en ambientes heterogéneos (Turchetto-Zolet et al., 2013). Estos patrones destacan la importancia de la dinámica de los procesos evolutivos y del mosaico de hábitats en los paisajes heterogéneos, los cuales permiten la persistencia de las especies a través de condiciones ambientales cambiantes (Turchetto-Zolet et al., 2013).

Los análisis demográficos aplicados a los datos cpSSRs en A. colubrina var. cebil permitieron el análisis del desarrollo histórico de los SDTFs en Argentina desde diferentes escalas. Desde una visión general, la fragmentación que condujo a la disyunción de estos bosques sería el principal evento demográfico-histórico y desde una visión particular de cada núcleo cada remanente fragmentario habría desarrollado su propia historia demográfica de manera independiente. De esta manera, puede concluirse que la distribución contemporánea de haplotipos cloroplásticos de A. colubrina var. cebil retiene evidencias de la fragmentación histórica entre los núcleos SDTFs, reflejada en la marcada estructuración genética cloroplástica, mientras que el núcleo Pedemontano Subandino retiene evidencias de una expansión posterior a su llegada a la región, reflejadas en la presencia de haplotipos raros, en tanto que el núcleo Misiones presenta estabilidad histórica dados los niveles de variación haplotípica contenidos.

\section{Agradecimientos}

Este trabajo fue financiado mediante los subsidios PICT 2014 N $^{\circ} 2352$ otorgado a M.E. Barrandeguy por la Agencia Nacional de Promoción Científica y Tecnológica (AGENCIA) y PICTO UNaM 2011 No0133 otorgado a M. V. García por la AGENCIA y la Universidad Nacional de Misiones (UNaM). Los autores agradecen al Consejo Nacional de Investigaciones Científicas y Técnicas (CONICET, Argentina). Se agradecen los comentarios y observaciones de dos revisores anónimos, que permitieron mejorar el manuscrito original.

\section{Biblografía}

AB'SÁBER, A.N. 1977. Os dominios morfoclimaticos na América do Sul: primeira aproximação. Geomorfologia 53: 1-23.

ALTSCHUL, S. von R. 1964. A taxonomic study of the genus Anadenanthera. Contrib. Gray Herb. Harvard Univ. 193: 3-65.

BANDELT, H. J., P. FORSTER \& A. RÖHL. 1999. Median-joining networks for inferring intraspecific phylogenies. Mol. Biol. Evol. 16: 37-48.

BARRANDEGUY, M. E., M. V. GARCÍA, K. PRINZ, R. RIVERAPOMAR \& R. FINKELDEY. 2014. Genetic structure of disjunct Argentinean populations of the subtropical tree Anadenanthera colubrina var. cebil (Fabaceae). Plant. Syst. Evol. 300: 1693-1705.

BECERRA, J. X. 2005 Timing the origin and expansion of the Mexican tropical dry forest. Proc. Natl. Acad. Sci. U.S.A. 102: 10919-10923.

CABRERA, A.L. 1976. Regiones Fitogeográficas Argentinas. 2da ed. Enciclopedia Argentina de Agricultura y Jardinería. ACME S.A.C.I., Buenos Aires.

CAETANO, S., D. PRADO, R. T. PENNINGTON, S. BECK, A. OLIVEIRA FILHO, R. SPICHIGER \& Y. NACIRI. 2008. The history of Seasonally Dry Tropical Forests in eastern South America: inferences from the genetic structure of the tree Astronium urundeuva (Anacardiaceae). Mol. Ecol. 17: 3147-3159.

CALONGA SOLÍS, V. C., M. E. BARRANDEGUY \& M.V. GARCÍA. 2014. Divergencia histórica en Anadenanthera colubrina var. cebil (Leguminosae) analizando una región intrónica del ADN cloroplástico. Bol. Soc. Argent. Bot. 49: 547-557.

CIALDELLA, A. 2000. Flora Fanerogámica Argentina. Proflora. Fabaceae Subfamilia Mimosoideae 67: 1-10.

COLLEVATTI, R. G., L. C. TERRIBILE, M. S. LIMARIBEIRO, J. C. NABOUT, G. OLIVEIRA, T. F. RANGEL, S. G. RABELO \& J. A. F. DINIZFILHO. 2012. A coupled phylogeographical and species distribution modelling approach recovers the 
demographical history of a Neotropical seasonally dry forest tree species. Mol. Ecol. 21: 5845-5863.

CORANDER, J., P. MARTTINEN \& S. MÄNTYNIEMI. 2006. Bayesian identification of stock mixtures from molecular marker data. Fish. Bull. 104: 550-558.

CORANDER, J., J. SIRÉN \& E. ARJAS. 2008a. Bayesian spatial modelling of genetic population structure. Comput. Stat. 23: 111-129.

CORANDER, J., P. MARTTINEN, J. SIRÉN \& J. TANG. 2008b. Enhanced Bayesian modelling in BAPS software for learning genetic structures of populations. BMC Bioinformatics 9: 1-14.

CRANDALL, K. A. \& A. R. TEMPLETON. 1993. Empirical tests of some predictions from coalescent theory with applications to intraspecific phylogeny reconstruction. Genetics 134: 959-69.

DAMUTH, J.E., R. W. FAIRBRIDGE. 1970. Equatorial Atlantic deep-sea arkosic sands and ice-age aridity in tropical South America. Geol. Soc. Am. Bull. 81: 189-206.

DAVIS, C.C., C.D. BELL, S. MATHEWS \& M.J. DONOGHUE. 2002. Laurasian migration explains Gondwanan disjunctions: evidence from Malpighiaceae. Proc. Natl. Acad. Sci. Biol. 99: 6833-6837.

DE OLIVEIRA, L.D.S.D., M.F. MORO, E.M. NIC LUGHADHA, F.R. MARTINS, A.L. DE MELO, H.-J. ESSER \& M.F. SALES. 2013. Hidden in the dry woods: Mapping the collection history and distribution of Gymnanthes boticario, a well-collected but very recently described species restricted to the dry vegetation of South America. Phytotaxa 97: 1-16.

DICK, C.W. \& M. HEUERTZ. 2008. The complex biogeographic history of a widespread tropical tree species. Evolution 62: 2760-2774.

EMERSON, B.C., PARADIS E., THEBAUD C. 2001. Revealing the demographic histories of species using DNA sequences. Trends Ecol. Evol. 16: 707-716.

EXCOFFIER, L., M. FOLL \& R. J. PETIT. 2009. Genetic Consequences of Range Expansions. Annu. Rev. Ecol. Evol. Syst. 40:481-501.

EXCOFFIER, L. \& H. LISCHER. 2010. Arlequin suite ver 3.5: A new series of programs to perform population genetics analyses under Linux and Windows. Mol. Ecol. Res. 10: 564-567.

FINKELDEY, R. \& H. HATTEMER .2007. Tropical forest genetics. Springer Verlag Edition, 315 pág.

FU, Y. X. 1997. Statistical tests of neutrality of mutations against population growth, hitchhiking and background selection. Genetics 147: 915-925.

GARCÍA, M. V., P. A. BALATTI \& M. J. ARTURI. 2007. Genetic variability in natural populations of Paspalum dilatatum Poir. analyzed by means of morphological traits and molecular markers. Genet. Resour. Crop. Ev. 54: 935-946.
GILÍ, F., X. AlBORNOZ, J. ECHEVERRÍA, M. GARCÍA, C. CARRASCO, F. MENESES \& H. M. NIEMEYER. 2016. Vilca, encuentro de miradas: antecedentes y herramientas para su pesquisa en contextos arqueológicos del área centro sur andina. Chungará (Arica). Artículo AOP. http://dx.doi. org/10.4067/S0717-73562016005000031

GILlET, E., D. GÖMÖRY \& L. PAULE. 2005. Measuring genetic variation within and among populations at marker loci. En Conservation and Management of forest genetic resources in Europe. Editado por Geburek, T. y J. Turok. Arbora Publishers 693 pág.

GONCALVES, A. L., M. E. BARRANDEGUY \& M. V. GARCÍA. 2013. Método económico de genotipificación por fluorescencia de SSRs en Anadenanthera colubrina var. cebil. J Basic Appl. Genet. XXIV (Supl 2013):107. Actas del XLII Congreso Argentino de Genética y III Reunión Regional SAG - NOA.

GONCALVES, A. L., M. E. BARRANDEGUY \& M. V. GARCÍA. 2014. Estructura y Representatividad Genética Cloroplástica en Poblaciones Naturales de Anadenanthera colubrina var. cebil (Leguminosae) de Argentina. Bol. Soc. Argent. Bot. 49: 235-245.

HAMRICK, J. L., D. A. MURAWSKI \& J. D. NASON. 1993. The influence of seed dispersal mechanisms on the genetic structure of tropical tree populations. Vegetatio 107: 281-297.

LAVIN, M., B.D. SCHRIRE, G.P. LEWIS, R.T. PENNINGTON, A. DELGADO-SALINAS, M. THULIN, C. HUGHES, A.B. MATOS \& M.F. WOJCIECHOWSKI. 2004. Metacommunity processes rather than continental tectonic history better explain geographically structured phylogenies in legumes. Philos. Trans. R. Soc. London B 359: 1509- 1522 .

LEIGH, J. W. \& D. BRYANT. 2015. PopART: fullfeature software for haplotype network construction. Methods Ecol. Evol. 6: 1110-1116.

MARTÍNEZ, O.G., M.E. BARRANDEGUY, M.V. GARCÍA, D.A. CACHARANI \&D.E. PRADO. 2013. Presencia de Anadenanthera colubrina var. colubrina (Fabaceae, Mimosoideae) en Argentina. Darwiniana, nueva serie 1: 279-288.

MAUTNER, T. 1997. The Penguin Dictionary of Philosophy. Penguin Books, Suffolk, 641 pp.

MAYLE, F. E. 2004. Assessment of the Neotropical dry forest refugia hypothesis in the light of palaeocological data and vegetation model simulations. J. Quat. Sci. 19: 713-720.

MOGNI, V. Y., L. J. OAKLEY \& D. E. PRADO. 2015. The distribution of woody legumes in neotropical dry forests: the Pleistocene Arc Theory 20 years on. Edinburgh J. Bot. 72: 35-60. 


\section{E. Barrandeguy et al. - Demografía histórica en A. colubrina var. cebil}

NAVASCUÉS, M., Z. VAXEVANIDOU, S. C. GONZÁLEZ-MARTÍNEZ, J. CLIMENT, L. GIL \& B. C. EMERSON. 2006. Chloroplast microsatellites reveal colonization and metapopulation dynamics in the Canary Island pine. Mol. Ecol. 15: 2691-2698.

NEI, M. 1987. Molecular evolutionary genetics. Columbia University Press, New York. 512 pág.

PEAKALL, R. \& P.E. SMOUSE. 2012. GenAlEx 6.5: genetic analysis in Excel. Population genetic software for teaching and research-an update. Bioinformatics 28: 2537-2539.

PENNINGTON, R. T., D. E. PRADO \& C. A. PENDRY. 2000. Neotropical seasonally dry forests and Quaternary vegetation changes. J. Biogeogr. 27: 261-273.

PENNINGTON, R. T., M. LAVIN, D. E. PRADO, C. A. PENDRY \& S. K. PELL. 2004. Historical climate change and speciation: neotropical seasonally dry forest plants show patterns of both Tertiary and Quaternary diversification. Philos. Trans. R. Soc. London 359:515-537.

PENNINGTON, R. T., M. LAVIN \& A. OLIVEIRAFILHO. 2009. Woody Plant Diversity, Evolution, and Ecology in the Tropics: Perspectives from Seasonally Dry Tropical Forests. Annu. Rev. Ecol. Evol. Syst. 40: 437-457.

PRADO, D.E. 1991. A critical evaluation of the floristic links between Chaco and Caatingas vegetation in South America. PhD Thesis, University of St. Andrews, Escocia.

PRADO, D.E. 2000. Seasonally dry forests of tropical South America: from forgotten ecosystems to a new phytogeographic unit. Edinburgh J. Bot. 57: 437-461.

PRADO, D. E. \& P. E. GIBBS. 1993. Patterns of species distributions in the dry seasonal forests of South America. Ann. Missouri Bot. Gard. 80: 902-927.

PYBUS, O. G. \& B. SHAPIRO. 2009. Natural selection and adaptation of molecular sequences. In The Phylogenetic Handbook, 2nd Edition (P Lemey, M Salemi, A Vandamme, editors) pp. 407-418. Cambridge University Press.

RAY, N., M. CURRAT \& L.EXCOFFIER. 2003. Intrademe molecular diversity in spatially expanding populations. Mol. Biol. Evol. 20: 76-86.

SCHUELKE, M. 2000. An economic method for the fluorescent labeling of PCR fragments. Nat. Biotechnol. 18: 233-234.

SCHRIRE, B.D., M. LAVIN, N.P. BARKER \& F. FOREST. 2009. Phylogeny of the tribe Indigofereae (Leguminosae-Papilionoideae): geographically structured more in succulent-rich and temperate settings than in grass-rich environments. Amer. $J$. Bot. 96: 816-852.
STEINER, J. J., C. J. POCKLEMBA, R. G. FJELLSTROM \& L. F. ELLIOT. 1995. A rapid one tube genomic DNA extraction process for PCR and RAPD analyses. Nucleic Acid Res. 23: 2569-2570.

TAJIMA, F. 1989. Statistical method for testing the neutral mutation hypothesis by DNA polymorphism. Genetics 123: 585-595.

TURCHETTO-ZOLET, A. C., F. PINHEIRO, F. SALGUEIRO \& C. PALMA-SILVA. 2013. Phylogeographical patterns shed light on evolutionary process in South America. Mol. Ecol. 22: 1193-1213.

VANZOLINI, P.E. 1963. Problemas faunísticos do cerrado. In: Simposio sobre o Cerrado (ed. Ferri MG). University of Sao Paulo Press, Sao Paulo 307-320.

WEIR, B. S. \& C. C. COCKERHAM. 1984. Estimating F-statistics for the analysis of population structure. Evolution 38: 1358-1370.

WEISING, K. \& R. GARDNER. 1999. A set of conserved PCR primers for the analysis of simple sequence repeat polymorphism in chloroplast genomes of dicotyledonous. Genome 42: 9-19.

WERNECK, F. P., G. C. COSTA, G. R. COLLI, D. E. PRADO \& J. W. SITES. 2011. Revisiting the historical distribution of Seasonally Dry Tropical Forests: new insights based on palaeodistribution modelling and palynological evidence. Glob. Ecol. Biogeogr. 20: 272-288.

WRIGHT, S. 1965. The interpretation of population structure by F-statistics with special regard to systems of mating. Evolution 19: 395-420.

YOUNG, A., T. BOYLE \& T. BROWN. 1996. The population genetic consequences of habitat fragmentation for plants. Trends Ecol. Evol. 11: 413-418.

ZHAN, Q. Q., J. F. WANG, X. GONG \& H. PENG. 2011. Patterns of chloroplast DNA variation in Cycas debaoensis (Cycadaceae): conservation implications. Conserv. Genet. 12: 959-970.

Recibido el 21 de marzo de 2016, aceptado el 6 de mayo de 2016. 
